PROCEEDINGS OF THE

AMERICAN MATHEMATICAL SOCIETY

Volume 138, Number 7, July 2010, Pages 2477-2487

S 0002-9939(10)10275-5

Article electronically published on March 4, 2010

\title{
HITTING TIME IN REGULAR SETS AND LOGARITHM LAW FOR RAPIDLY MIXING DYNAMICAL SYSTEMS
}

\author{
STEFANO GALATOLO
}

(Communicated by Bryna Kra)

\begin{abstract}
We prove that if a system has superpolynomial (faster than any power law) decay of correlations (with respect to Lipschitz observables), then the time $\tau\left(x, S_{r}\right)$ is needed for a typical point $x$ to enter for the first time a set $S_{r}=\{x: f(x) \leq r\}$ which is a sublevel of a Lipschitz function $f$ scales as $\frac{1}{\mu\left(S_{r}\right)}$ i.e.,

$$
\lim _{r \rightarrow 0} \frac{\log \tau\left(x, S_{r}\right)}{-\log r}=\lim _{r \rightarrow 0} \frac{\log \mu\left(S_{r}\right)}{\log r} .
$$

This generalizes a previous result obtained for balls. We will also consider relations with the return time distributions, an application to observed systems and to the geodesic flow in negatively curved manifolds.
\end{abstract}

\section{INTRODUCTION AND STATEMENT OF RESULTS}

Let $(X, T, \mu)$ be an ergodic system on a metric space $X$ and fix a point $x_{0} \in X$. For $\mu$-almost every $x \in X$, the orbit of $x$ gets closer and closer to $x_{0}$, entering (sooner or later) in each positive measure neighborhood of the target point $x_{0}$.

For several applications it is useful to quantify the speed of approach of the orbit of $x$ to $x_{0}$. In the literature this has been done in several ways, with more or less precise estimations or considering different kinds of target sets.

A general approach is to consider a family of sets $S_{r}$ indexed by a real parameter $r$ containing $x_{0}$ and give an estimate for the time needed for the orbit of a point $x$ to enter in $S_{r}$,

$$
\tau\left(x, S_{r}\right)=\min \left\{n \in \mathbb{N}^{+}: T^{n}(x) \in S_{r}\right\} .
$$

If $X$ is a metric space, the most natural choice is to take $S_{r}=B_{r}\left(x_{0}\right)$ (the ball of radius $r$ ). In this case several estimates are known for the behavior of $\tau\left(x, S_{r}\right)$ as $r \rightarrow 0$. For example, if the system has fast decay of correlations or is a circle rotation with generic arithmetical properties, then for a.e. $x$

$$
\lim _{r \rightarrow 0} \frac{\log \tau\left(x, B_{r}\left(x_{0}\right)\right)}{-\log r}=d_{\mu}\left(x_{0}\right) .
$$

Received by the editors June 18, 2009, and, in revised form, October 12, 2009. 2010 Mathematics Subject Classification. Primary 37A25, 37C45, 37D40, 37A99.

Key words and phrases. Logarithm law, hitting time, decay of correlations, dimension, return time distribution.

(C)2010 American Mathematical Society Reverts to public domain 28 years from publication 
Similar results also hold for generic interval exchange transformations (see [6, 9, 13, 15]). It is worth remarking that in this case $\tau\left(x, B_{r}\left(x_{0}\right)\right) \sim r^{-d_{\mu}} \sim \frac{1}{\mu\left(S_{r}\right)}$, 1] as is natural to expect in a system having "stochastic" behavior.

On the other hand it is worth remarking that there are mixing systems (having particular arithmetical properties and slow decay of correlations) for which $\liminf _{r \rightarrow 0} \frac{\log \tau\left(x, B_{r}\left(x_{0}\right)\right)}{-\log r}=\infty>d_{\mu}\left(x_{0}\right)$ (see [7]).

In some cases, even if $X$ is a metric space it is interesting to look at different target sets: for example, considering a tubular neighborhood of a "singular set" and asking how much time we need to approach the singular set at a distance $r$ (see [4]).

Another interesting situation is when $X$ has a local product structure and one is interested in approaching only some coordinates, for example, when $X$ is a tangent bundle of a manifold $M$ and the dynamics is given by the geodesic flow. Here one can be interested in approaching a given target in $M$ or in approaching the "point at infinity", giving no importance to the other coordinates (see e.g. [18, 21]). Other examples are given if one considers the structure given by stable and unstable directions ([2]). Another particularly interesting case where a set other than balls becomes interesting is the case of observed systems (see section 3.2 and [20] for a similar point of view in quantitative recurrence).

We remark that in some of the cited papers the problem of estimating the speed of approach to a target point is not always stated in the above form, considering the hitting time to a neighborhood of the target. In such papers, the authors consider the behavior of the distance from a typical orbit to the target point (see (3.8)) or state some kind of dynamical Borel-Cantelli lemma (see e.g. 2, [3, 11], which can give a slightly more precise estimation for typical hitting times in small sets than the one given in (1.2)); general relations between these approaches can be found in [7] and [10].

In this paper we take the point of view of (1.2) and consider target sets of the form $S_{r}=\{x \in X, f(x) \leq r\}$, where $f$ is a Lipschitz function. The main result (see Theorem 3.2 for a precise statement) is a generalization of the one given in [6] for this more general family of sets: if the system has superpolynomial decay of correlations with respect to Lipschitz observables, then the power law behavior of the hitting time $\tau\left(x, S_{r}\right)$ (as defined in (1.1)) in the sets $S_{r}$ satisfies a "logarithm law" of the form

$$
\lim _{r \rightarrow 0} \frac{\log \tau\left(x, S_{r}\right)}{-\log r}=d_{\mu}(f)
$$

where $d_{\mu}(f)=\lim _{r \rightarrow 0} \frac{\log \mu\left(S_{r}\right)}{\log (r)}$, generalizing the formula for the local dimension which is in the right hand side of (1.2).

We will also give a similar statement for observed systems (see section 3.2), an application (see section 3.1) to geodesic flows on negatively curved manifolds and prove some relations with limit return time statistic (see Theorem 4.1) in the sets $S_{r}$.

\footnotetext{
${ }^{1}$ Where $\sim$ stands for some kind of asymptotical equivalent behavior. In this context $a(r) \sim b(r)$ if $\lim _{r \rightarrow 0} \frac{\log a(r)}{\log r}=\lim _{r \rightarrow 0} \frac{\log b(r)}{\log r}$.
} 


\section{Setting And BAsic Results}

Let $f$ be a Borel measurable function such that $f \geq 0$ on $X$. Let us consider sublevel sets $S_{r}=\{x \in X, f(x) \leq r\}$. Let us define an indicator for the power law behavior of the hitting time to the set $S_{r}$ as $r \rightarrow 0$ :

$$
\bar{R}(x, f)=\limsup _{r \rightarrow 0} \frac{\log \tau\left(x, S_{r}\right)}{-\log (r)}, \underline{R}(x, f)=\liminf _{r \rightarrow 0} \frac{\log \tau\left(x, S_{r}\right)}{-\log (r)} .
$$

In this way if $\bar{R}(x, f)=\underline{R}(x, f)=R(x, f)$, then $\tau\left(x, S_{r}\right) \sim r^{-R(x, f)}$ for small $r$. Comparing this notation with that given in other papers, we remark that the indicator $R(x, y)$ considered [ [6] is obtained from $R(x, f)$ when $f(x)=d(x, y)$ and the indicator $R(x)$ of [1] is obtained as a further special case when $x=y$.

Let us recall the definition of local dimension of a measure on a metric space. If $X$ is a metric space and $\mu$ is a measure on $X$, the local dimension of $\mu$ at $x$ is defined as $d_{\mu}(x)=\lim _{r \rightarrow 0} \frac{\log (\mu(B(x, r)))}{\log (r)}$ (when the limit exists). The upper local dimension at $x \in X$ is defined as $\bar{d}_{\mu}(x)=\limsup _{r \rightarrow 0} \frac{\log (\mu(B(x, r)))}{\log (r)}$, and the lower local dimension $\underline{d}_{\mu}(x)$ is defined in an analogous way by replacing limsup with lim inf. If $\bar{d}_{\mu}(x)=\underline{d}_{\mu}(x)=d$ almost everywhere the measure is called exact dimensional. In this case many notions of dimension of a measure will coincide (see e.g. [19]). By analogy with the definition of local dimension let us consider

$$
\bar{d}_{\mu}(f)=\limsup _{r \rightarrow 0} \frac{\log \mu\left(S_{r}\right)}{\log (r)}, \underline{d}_{\mu}(f)=\liminf _{r \rightarrow 0} \frac{\log \mu\left(S_{r}\right)}{\log (r)} .
$$

Between the indicators defined in (2.1) and (2.2) there is a general relationship, which follows by a direct application of the classical Borel-Cantelli lemma:

Proposition 1. Let $(X, T, \mu)$ be a measure preserving transformation and $f$ be as above. Then

$$
\bar{R}(x, f) \geq \bar{d}_{\mu}(f), \underline{R}(x, f) \geq \underline{d}_{\mu}(f)
$$

$\mu$-a.e.

Before proving the above proposition we make an elementary remark on the behavior of real sequences which will often be used below.

Lemma 2.1. Let $r_{n}$ be a decreasing sequence such that $r_{n} \rightarrow 0$. Suppose that there is a constant $c>0$ satisfying $r_{n+1}>c r_{n}$ eventually as $n$ increases. Let $\tau_{r}: \mathbb{R} \rightarrow \mathbb{R}$ be decreasing. Then $\liminf n_{n \rightarrow \infty} \frac{\log \tau_{r_{n}}}{-\log r_{n}}=\liminf _{r \rightarrow 0} \frac{\log \tau_{r}}{-\log r}$ and $\limsup \sup _{n \rightarrow \infty} \frac{\log \tau_{r_{n}}}{-\log r_{n}}=\limsup \sup _{r \rightarrow 0} \frac{\log \tau_{r}}{-\log r}$.

Proof of Proposition 1. First we prove $\underline{R}(x, f) \geq \underline{d}_{\mu}(f)$. Let us consider the set of $x$, where $\underline{R}(x, f)<\underline{d}_{\mu}(f)$; we will prove that this set has zero measure. By the above lemma we will consider a sequence of radii $r_{k}$ of the form $r_{k}=2^{-k}$. Let us consider $d<\underline{d}_{\mu}(f)$; then we eventually have that $\mu\left(S_{2^{-k}}\right) \leq 2^{-d k}$. Let us consider $d^{\prime}<d$ and

$$
A\left(d^{\prime}\right)=\left\{x \in X \mid \underline{R}(x, f) \leq d^{\prime}\right\} .
$$

By the definition of $\underline{R}(x, f)$, it holds that for each $m$

$$
A\left(d^{\prime}\right) \subset \bigcup_{n \geq m} \bigcup_{i \leq 2^{n\left(\frac{d+d^{\prime}}{2}\right)}} T^{-i}\left(S_{2^{-n}}\right),
$$


but $\mu\left(\bigcup_{i \leq 2^{n\left(\frac{d+d^{\prime}}{2}\right)}} T^{-i}\left(S_{2^{-n}}\right)\right) \leq 2^{n\left(\frac{d+d^{\prime}}{2}\right)} \times 2^{-d n}$ and $n\left(\frac{d+d^{\prime}}{2}\right)-d n<0$. Hence this sequence of sets has summable measure and by the classical Borel-Cantelli lemma $\mu\left(A\left(d^{\prime}\right)\right)=0$, we prove the first inequality.

Now we prove $\bar{R}(x, f) \geq \bar{d}_{\mu}(f)$. Again, we can suppose $r_{k}$ to be of the form $r_{k}=2^{-k}$. Suppose $d^{\prime}<\bar{d}_{\mu}(f)$; let us consider

$$
A\left(d^{\prime}\right)=\left\{x \in X \mid \bar{R}(x, f)<d^{\prime}\right\} .
$$

If $0<d^{\prime}<d<\bar{d}_{\mu}(f)$, then there is a sequence $n_{k}$ such that

$$
\mu\left(S_{2^{-n_{k}}}\right)<2^{-d n_{k}} \text { for each } k \text {. }
$$

On the other side, for each $x \in A\left(d^{\prime}\right)$ the relation $\tau\left(x, S_{2^{-n}}\right)<2^{\frac{d+d^{\prime}}{2} n}$ must eventually hold. Let us consider

$$
C(m)=\left\{x \in A\left(d^{\prime}\right) \mid \forall n \geq m, \tau\left(x, S_{2^{-n}}\right)<2^{\frac{d+d^{\prime}}{2} n}\right\} .
$$

This is an increasing sequence of sets "converging" to $A$. If we prove that

$$
\liminf _{m \rightarrow \infty} \mu(C(m))=0,
$$

the statement is proved. By the definition of $C(m)$ we see that

$$
C\left(n_{k}\right) \subset \bigcup_{i \leq 2^{\frac{d+d^{\prime}}{2}} n_{k}} T^{-i}\left(S_{2^{-n_{k}}}\right) .
$$

The latter is made of $2^{\frac{d+d^{\prime}}{2} n_{k}}$ sets, whose measure can be estimated by (2.5), because $T$ is measure preserving. Then $\mu\left(C\left(n_{k}\right)\right) \leq 2^{\frac{d+d^{\prime}}{2} n_{k}} \times 2^{-d n_{k}}$ and $\mu\left(C\left(n_{k}\right)\right)$ goes to 0 as $k \rightarrow \infty$.

\section{FAST MIXING SYSTEMS}

As is well known, in a mixing system we have $\mu\left(A \cap T^{-n}(B)\right) \rightarrow \mu(A) \mu(B)$ for each measurable set $A, B$. The speed of convergence of the above limit can be arbitrarily slow (depending on $T$ but also on the shape of the sets $A, B$ ). In many systems, however, the speed of convergence can be estimated for sets having some regularity.

Let us remark that considering $1_{A}(x)=\left\{\begin{array}{l}1 \text { if } x \in A, \\ 0 \text { if } x \notin A\end{array}\right.$ the mixing condition becomes $\int 1_{B} \circ T^{n} 1_{A} d \mu \rightarrow \int 1_{A} d \mu \int 1_{B} d \mu$.

Definition 3.1. Let $\phi, \psi: X \rightarrow \mathbb{R}$ be Lipschitz observables on $X$. A system $(X, T, \mu)$ is said to have superpolynomial decay of correlations with respect to Lipschitz observables if, for each such $\phi, \psi$,

$$
\left|\int \phi \circ T^{n} \psi d \mu-\int \phi d \mu \int \psi d \mu\right| \leq\|\phi\|\|\psi\| \Phi(n),
$$

with $\Phi$ having superpolynolmial decay; i.e. $\lim n^{\alpha} \Phi(n)=0, \forall \alpha>0$.

Here \|\| is the Lipschitz norm. This is one of the weakest requirements on the space of observables. Decay of correlations with respect to Holder observables implies it. In the remaining part of this section we will prove the following result. 
Theorem 3.2. If $f: X \rightarrow \mathbb{R}^{+}$is $\ell$-Lipschitz, the system has superpolynomial decay of correlations, as above, and $\underline{d}_{\mu}(f)=\bar{d}_{\mu}(f)=d_{\mu}(f)<\infty$, then for a.e. $x$ it holds that

$$
R(x, f)=d_{\mu}(f) .
$$

Before proving the theorem we will need some preliminary lemmas: the first is technical and allows us to use decay of correlation to estimate the measure of certain intersections of sublevel sets.

Lemma 3.3. Let $r_{n} \searrow 0$ and let $S_{r_{k}}$ be a sequence of nested sets which are sublevel sets of an $\ell$-Lipschitz function as above. Let $A_{k}=T^{-k}\left(S_{r_{k}}\right)$ and let us write $A_{-1}=X$. If $(X, T, \mu)$ is a system satisfying Definition 3.1, then there is $N$ such that when $k>j>N$,

$$
\mu\left(A_{k} \cap A_{j}\right) \leq \mu\left(A_{k-1}\right) \mu\left(A_{j-1}\right)+\frac{4 \ell^{2} \Phi(k-j)}{\left(r_{k-1}-r_{k}\right)\left(r_{j-1}-r_{j}\right)} .
$$

Proof. Let

$$
f_{n}(x)= \begin{cases}\frac{r_{n-1}-f(x)}{r_{n-1}-r_{n}} & \text { if } x \in S_{r_{n-1}}-S_{r_{n}} \\ 1 & \text { if } x \in S_{r_{n}} \\ 0 & \text { if } x \notin S_{r_{n-1}}\end{cases}
$$

these are $\frac{\ell}{r_{n-1}-r_{n}}$-Lipschitz functions with support in $S_{r_{n-1}}$. Notice that $\mu\left(S_{r_{n}}\right) \leq$ $\int f_{n}(x) d \mu \leq \mu\left(S_{r_{n-1}}\right)$. The Lipschitz norm is such that $\left\|f_{k}\right\|_{L i p} \leq \frac{\ell}{r_{n-1}-r_{n}}+1$. If $N$ is large enough such that $r_{n-1}-r_{n} \leq \ell, \forall n \geq N$, then $\left\|f_{k}\right\|_{L i p} \leq \frac{2 \ell}{r_{n-1}-r_{n}}$. Let $k>j>N$. Since $\mu$ is preserved,

$$
\mu\left(A_{k} \cap A_{j}\right)=\mu\left(T^{-k+j}\left(S_{r_{k}}\right) \cap S_{r_{j}}\right) \leq \int f_{k} \circ T^{k-j} f_{j} d \mu .
$$

By decay of correlations

$$
\begin{aligned}
\int f_{k} \circ T^{k-j} f_{j} d \mu & \leq \int f_{k} d \mu \int f_{j} d \mu+\left\|f_{k}\right\|_{L i p}\left\|f_{j}\right\|_{L i p} \Phi(k-j) \\
& \leq \mu\left(A_{k-1}\right) \mu\left(A_{j-1}\right)+\left\|f_{k}\right\|_{L i p}\left\|f_{j}\right\|_{L i p} \Phi(k-j),
\end{aligned}
$$

which gives the statement.

The second lemma that we will use is a sort of dynamical Borel-Cantelli lemma for systems having fast decay of correlations. This was proved in [6] (Lemma 7).

Lemma 3.4. Let $S_{k}$ be a decreasing sequence of measurable sets such that

$$
\liminf _{k \rightarrow \infty} \frac{\log \left(\sum_{0}^{k} \mu\left(S_{k}\right)\right)}{\log (k)}=z>0 .
$$

Let $A_{k}=T^{-k}\left(S_{k}\right)$ and let us suppose that the system is such that when $k>j$

$$
\mu\left(A_{k} \cap A_{j}\right) \leq \mu\left(A_{k-1}\right) \mu\left(A_{j-1}\right)+k^{c_{1}} j^{c_{2}} \Phi(k-j)
$$

with $\Phi$ having superpolynomial decay and $c_{1}, c_{2} \geq 0$. Then posing

$$
Z_{k}(x)=\sum_{i=0}^{k} 1_{A_{i}}(x)
$$

we have $\frac{Z_{k}}{E\left(Z_{k}\right)} \rightarrow 1$ in the $L^{2}$ norm and almost everywhere. 
We remark that, since the set sequence is decreasing, the condition $\mu\left(A_{k} \cap A_{j}\right) \leq$ $\mu\left(A_{k-1}\right) \mu\left(A_{j-1}\right)+\ldots$ is slightly more relaxed than $\mu\left(A_{k} \cap A_{j}\right) \leq \mu\left(A_{k}\right) \mu\left(A_{j}\right)+\ldots$. This is a technical point which will allow us to use Lemma 3.3 without further technical complications and apply Lemma 3.4 to the sequence $S_{r_{k}}$. This will allow us to prove the main result.

Proof of Theorem 3.2. For simplicity of notation let us set $d=d_{\mu}(f)$. Let us prove $\bar{R}(x, f) \leq d$ for almost every $x$. We recall that this implies $\underline{R}(x, f) \leq d$ and the opposite inequalities come from Proposition 1. Let us consider $0<\beta<\frac{1}{d}$, the sequence $r_{k}=k^{-\beta}$ and set $S_{k}=S_{r_{k}}$ (we remark that if the result is proved for such a subsequence, then it holds for all subsequences; see Lemma 2.1). For each small $\epsilon<\beta^{-1}-d$, eventually $\mu\left(S_{k}\right) \geq\left(r_{k}\right)^{d+\epsilon}=k^{-\beta(d+\epsilon)}$; and if $k$ is big enough, then $\sum_{0}^{k} \mu\left(S_{k}\right) \geq C k^{1-\beta(d+\epsilon)}$ for some $C>0$. Since $\epsilon$ is arbitrary we have

$$
\liminf _{k \rightarrow \infty} \frac{\log \left(\sum_{0}^{k} \mu\left(S_{k}\right)\right)}{\log (k)} \geq 1-\beta(d+\epsilon)>0 .
$$

Moreover, there is $C>0$ s.t. $\frac{1}{C}<\frac{r_{k-1}-r_{k}}{k^{-\beta-1}}<C$. Hence we can apply Lemma 3.3 to the sequence $S_{k}$ and Lemma 3.4 to the sequence $\tilde{S}_{k}=\left\{\begin{array}{c}X \text { if } k \leq N \\ S_{k} \text { if } k>N\end{array}\right.$, where $N$ is the one given in Lemma 3.3. We then obtain that the sequence $Z_{k}(x)=$ $\sum_{i=0}^{k} 1_{T^{-i}\left(S_{i}\right)}(x)$ satisfies $\lim _{n \rightarrow \infty} \frac{Z_{n}}{E\left(Z_{n}\right)}=1, \mu$-almost everywhere.

Let us now consider $\epsilon^{\prime}>0$ such that $\beta\left(d+\epsilon^{\prime}\right)>1$ for $\beta$ as above, near to $\frac{1}{d}$ (recall that $\beta d<1$ ). Moreover, let us consider $\varepsilon>0$ so small that $\beta(d+\varepsilon)<1$ and $\beta\left(d+\epsilon^{\prime}\right)-\frac{1-\beta(d-\varepsilon)}{1-\beta(d+\varepsilon)}>0$. Let us consider $x$ such that $\bar{R}(x, f)>d+\epsilon^{\prime}$; then for infinitely many $n, \tau\left(x, S_{n}\right)>n^{\beta\left(d+\epsilon^{\prime}\right)}$. Then

$$
x \notin \bigcup_{0 \leq i \leq\left\lfloor n^{\beta\left(d+\epsilon^{\prime}\right)}\right\rfloor} T^{-i}\left(S_{n}\right)
$$

and in particular (remarking that since $\beta\left(d+\epsilon^{\prime}\right)>1$, then $n \leq n^{\beta\left(d+\epsilon^{\prime}\right)}$ )

$$
x \notin \bigcup_{n \leq i \leq\left\lfloor n^{\beta\left(d+\epsilon^{\prime}\right)}\right\rfloor} T^{-i}\left(S_{n}\right) \supset \bigcup_{n \leq i \leq\left\lfloor n^{\beta\left(d+\epsilon^{\prime}\right)}\right\rfloor} T^{-i}\left(S_{i}\right),
$$

which implies that there is a sequence $n_{i}$ such that $Z_{n_{i}}(x)=Z_{\left\lfloor n_{i}^{\beta\left(d+\epsilon^{\prime}\right)}\right\rfloor}(x)$ for each $i$. Now let us consider $E\left(Z_{n_{i}}\right)$ and $E\left(Z_{\left.n_{i}^{\beta\left(d+\epsilon^{\prime}\right)}\right]}\right)$. By the definition of $d=d_{\mu}(f)$, when $i$ is large enough

$$
i^{-\beta(d+\varepsilon)}<\mu\left(S_{i}\right)<i^{-\beta(d-\varepsilon)} .
$$

Then there are constants $k_{1}$ and $k_{2}$ such that when $n$ is large enough, $k_{1} n^{1-\beta(d+\varepsilon)}<$ $E\left(Z_{n}\right)<k_{2} n^{1-\beta(d-\varepsilon)}$. From this we have that if $i$ is large enough,

$$
\begin{aligned}
& \frac{E\left(Z_{n_{i}}\right)}{E\left(Z_{\left\lfloor n_{i}^{\beta\left(d+\epsilon^{\prime}\right)}\right\rfloor}\right)} \leq \frac{k_{2} n_{i}^{1-\beta(d-\varepsilon)}}{k_{1}\left\lfloor n_{i}^{\beta\left(d+\epsilon^{\prime}\right)}\right\rfloor^{(1-\beta(d+\varepsilon))}} l \\
& \leq C_{2} n_{i}^{(1-\beta(d-\varepsilon))-\beta\left(d+\epsilon^{\prime}\right)(1-\beta(d+\varepsilon))}
\end{aligned}
$$


for some $C_{2}>0$. By the assumptions on $\varepsilon,(1-\beta(d-\varepsilon))-\beta\left(d+\epsilon^{\prime}\right)(1-\beta(d+\varepsilon))=$ $(1-\beta(d+\varepsilon))\left(\frac{1-\beta(d-\varepsilon)}{1-\beta(d+\varepsilon)}-\beta\left(d+\epsilon^{\prime}\right)\right)<0 ;$ hence

$$
\lim _{i \rightarrow \infty} \frac{E\left(Z_{n_{i}}\right)}{E\left(Z_{\left\lfloor n_{i}^{\beta\left(d+\epsilon^{\prime}\right)}\right\rfloor}\right)}=0 .
$$

Since $n_{i}$ was chosen such that $Z_{n_{i}}(x)=Z_{\left\lfloor n_{i}^{\beta\left(\bar{d}_{\mu}(f)+\epsilon^{\prime}\right)}\right\rfloor}(x)$, this implies that

$$
\frac{Z_{n_{i}}(x)}{E\left(Z_{n_{i}}\right)} \frac{E\left(Z_{\left\lfloor n_{i}^{\beta\left(d+\epsilon^{\prime}\right)}\right\rfloor}\right)}{Z_{\left\lfloor n_{i}^{\beta\left(d+\epsilon^{\prime}\right)}\right\rfloor}(x)}=\frac{\left.E\left(Z n_{i}^{\beta\left(d+\epsilon^{\prime}\right)}\right\rfloor\right)}{E\left(Z_{n_{i}}\right)} \rightarrow \infty
$$

as $i$ increases. Then it is not possible that $\lim _{n \rightarrow \infty} \frac{Z_{n}(x)}{E\left(Z_{n}(x)\right)}=1$. This implies that $\bar{R}(x, f)>d+\epsilon^{\prime}$ on a zero measure set. Finally, since $\epsilon^{\prime}$ can be chosen to be arbitrarily small, we have the statement.

3.1. Geodesic flow and its time one map. As a simple application of the main theorem (Theorem 3.2), we give a logarithm law for the time-one map of the geodesic flow in a negatively curved manifold. This result is in some sense a discrete time version of the main result of [18. We remark that since we only need an estimate for the decay of correlation, we can apply the deep results available for this kind of flow and consider manifolds with (variable) negative curvature instead of constant curvature. We will use the following result from [17]:

Theorem 3.5. The geodesic flow $T^{t}$ of a $C^{4}$ compact manifold with strictly negative curvature is exponentially mixing with respect to Hölder observables: there exists $C$ and $\sigma>0$ such that

$$
\left|\int \phi \circ T^{t} \psi d \mu-\int \phi d \mu \int \psi d \mu\right| \leq C\|\phi\|_{\alpha}\|\psi\|_{\alpha} e^{-\sigma t}
$$

Theorem 3.2 hence gives

Proposition 2. Let $M$ be a $C^{4}$, compact manifold of dimension $d$ with strictly negative curvature and $T^{1} M$ be its unitary tangent bundle. Let $\pi: T^{1} M \rightarrow M$ be the canonical projection. If $T$ is the time 1 map of the geodesic flow, $\mu$ the Liouville measure on $T^{1} M$, and dist() the Riemannian distance on $M$, then for each $p \in M$,

$$
\limsup _{n \rightarrow \infty} \frac{-\log \operatorname{dist}\left(p, \pi\left(T^{n} x\right)\right)}{\log n}=\frac{1}{d}
$$

holds for almost each $x \in T^{1} M$.

Proof. By the above theorem, the time 1 map associated to the flow has exponential decay of correlations for Hölder observables and hence for Lipschitz ones too. Let us consider the function $f: T^{1} M \rightarrow \mathbb{R}$ given by $f(x)=\operatorname{dist}(\pi(x), p)$. Since the Liouville measure is absolutely continuous, with density bounded away from zero, the associated sets $S_{r}$ are such that $\frac{\log \mu\left(S_{r}\right)}{\log (r)} \rightarrow d$. Then by Theorem 3.2

$$
\lim _{r \rightarrow 0} \frac{\log \tau\left(x, S_{r}\right)}{-\log (r)}=d .
$$


Let $d_{n}(x, p)=\min _{i \leq n} \operatorname{dist}\left(\pi\left(T^{i}(x)\right), p\right)$. Since $x$ can vary in a full measure set, we can suppose $\operatorname{dist}\left(\pi\left(T^{i}(x)\right), p\right) \neq 0$ for each $i \in \mathbb{N}$. Let us suppose that for $n$ large enough, $d_{n}=n^{-\alpha(n)}$. Since $\lim _{n \rightarrow 0} \frac{\log \tau\left(x, S_{n}-\alpha(n)\right)}{-\log \left(n^{-\alpha(n)}\right)}=d$, then $\forall \epsilon$, if $n$ is large enough,

$$
n^{\alpha(n) d-\alpha(n) \epsilon} \leq \tau\left(x, S_{n^{-\alpha(n)}}\right) \leq n^{\alpha(n) d+\alpha(n) \epsilon},
$$

but $\tau\left(x, S_{n^{-\alpha(n)}}\right) \leq n$; hence $n^{\alpha(n) d-\alpha(n) \epsilon} \leq n, \alpha(n) d-\alpha(n) \epsilon \leq 1$ and then $\alpha(n) \leq \frac{1}{d-\epsilon}$. This implies that $\limsup _{n \rightarrow \infty} \frac{-\log \frac{S}{d_{n}}\left(p, \pi\left(T^{n} x\right)\right)}{\log n} \leq \frac{1}{d}$. On the other hand there are infinitely many $n$ such that $\tau\left(x, S_{n^{-\alpha(n)}}\right)=n$, and hence this is less than or equal to $n^{\alpha(n) d+\alpha(n) \epsilon}$. With the same calculation as above we have the statement.

3.2. Observed systems. An important case where hitting time for sets different from balls becomes interesting and our approach very natural is the case of observed systems. Here the behavior of the system $(X, d, T, \mu)$ is observed through a measurable function $F: X \rightarrow Y$ ( $Y$ is supposed to be a metric space with distance $\left.d^{\prime}\right)$, and we look to the time needed for $F\left(T^{n} x\right)$ to approach $F\left(x_{0}\right)$ (in [20] something similar was done for quantitative recurrence indicators).

The function naturally induces a measure $F^{*}(\mu)$ on $Y$, defined as $F^{*}(\mu)[A]=$ $\mu\left(F^{-1}(A)\right)$ for each measurable set $A \subseteq Y$. We can then consider the local dimension of the induced measure $d_{F^{*}(\mu)}$ of $F^{*}(\mu)$ and the observed hitting times:

$$
\tau_{r}^{F}\left(x, x_{0}\right)=\min \left\{k \in \mathbb{N}^{+}, F\left(T^{k}(x)\right) \in B_{r}\left(F\left(x_{0}\right)\right)\right\} .
$$

Proposition 3. Let us consider $f: X \rightarrow \mathbb{R}$ defined by $f(x)=d^{\prime}\left(F(x), F\left(x_{0}\right)\right)$. Then for each $x \in X$,

$$
\begin{aligned}
d_{F^{*}(\mu)}\left(F\left(x_{0}\right)\right) & =d_{\mu}(f), \\
\tau_{r}^{F}\left(x, x_{0}\right) & =\tau\left(x, S_{r}\right),
\end{aligned}
$$

where $S_{r}=\{x \in X, f(x) \leq r\}$ as in (2.2) and $d_{\mu}(f)$ is defined as in (2.2).

Proof. The proof is straightforward from the definitions

$$
\begin{aligned}
d_{F^{*}(\mu)}\left(F\left(x_{0}\right)\right) & =\lim _{r \rightarrow 0} \frac{\log \left(F^{*}(\mu)\left[B_{r}\left(F\left(x_{0}\right)\right)\right]\right)}{\log r}=\lim _{r \rightarrow 0} \frac{\log \left(\mu\left(F^{-1}\left(B_{r}\left(F\left(x_{0}\right)\right)\right)\right)\right)}{\log r} \\
& =\lim _{r \rightarrow 0} \frac{\log \mu\left(\left\{x \in X, d^{\prime}\left(F(x), F\left(x_{0}\right)\right) \leq r\right\}\right)}{\log r}=\lim _{r \rightarrow 0} \frac{\log \mu\left(S_{r}\right)}{\log r}=d_{\mu}(f) ;
\end{aligned}
$$

moreover,

$$
\tau_{r}^{F}\left(x, x_{0}\right)=\min \left\{k \in \mathbb{N}^{+}, F\left(T^{k}(x)\right) \in B_{r}\left(F\left(x_{0}\right)\right)\right\}=\tau\left(x, S_{r}\right) .
$$

This directly gives the following corollary of Theorem 3.2 for observed systems.

Corollary 1. If we consider an observed system as above, $F: X \rightarrow Y$ is Lipschitz, the system has superpolynomial decay of correlations, $d_{F^{*}(\mu)}\left(F\left(x_{0}\right)\right)$ exists and it is finite as in Theorem 3.2, then

$$
\lim _{r \rightarrow 0} \frac{\log \tau_{r}^{F}\left(x, x_{0}\right)}{-\log (r)}=d_{F^{*}(\mu)}\left(F\left(x_{0}\right)\right)
$$

$\mu$-a.e. 
The following theorem from 20] ensures the existence of the local dimension for a class of interesting examples of observed systems.

Theorem 3.6. Let $F: \mathbb{R}^{m} \rightarrow \mathbb{R}^{n}$ be a $C^{\infty}$ function and let $\mu$ be an absolutely continuous measure on $\mathbb{R}^{m}$. Then $d_{F^{*}(\mu)}$ exists and belongs to the set $\{0,1, \ldots, \min (m, n)\}$ almost everywhere. More precisely, $d_{F^{*}(\mu)}(f(x))=\operatorname{rank}\left(d_{x} F\right)$ for $\mu$-almost every $x \in \mathbb{R}^{M}$.

\section{A relation with Return time statistics}

The return time statistics is a widely studied feature of dynamics (see e.g. 16, 1 and the references therein) and has links with other subjects, such as the extreme value theory ([5]). Let us consider a Lipschitz function $f$, the above sets $S_{r}=\{x$ : $f(x) \leq r\}$ and let us suppose that $\mu\left(S_{0}\right)=0$ and $\mu\left(S_{r}\right) \neq 0$ for $r>0$. We will consider the statistical distribution of return times in these sets. We say that the return time statistics of $(X, T)$ converges to $g$ for the sets $S_{r}$ if

$$
\lim _{r \rightarrow 0} \frac{\mu\left(\left\{x \in S_{r}, \tau\left(x, S_{r}\right) \geq \frac{t}{\mu\left(S_{r}\right)}\right\}\right)}{\mu\left(S_{r}\right)}=g(t) .
$$

If $g(t)=e^{-t}$ we say that the system has exponential return time limit statistics. Such statistics can be found in several systems with some hyperbolic behavior in some class of decreasing sets; however other limit distributions are possible. The following theorem shows that if the logarithm law does not hold, then the return time statistic has a trivial limit.

Theorem 4.1. If $(X, T, \mu)$ preserves the finite measure $\mu$ and

$$
\underline{R}(x, f)>\bar{d}_{\mu}(f)
$$

a.e., then the system has trivial limit return time statistic in the sets $S_{r}$. That is, the limit in (4.1) exists for each $t$ and $g(t)=0$.

Let us consider the set

$$
C_{l, r}=\left\{x \in S_{r}, \tau\left(x, S_{r}\right)>l \mu\left(S_{r}\right)^{-1}\right\} .
$$

We remark that if the return time statistic converges to $g$ as above, then $\lim _{r \rightarrow 0} \frac{\mu\left(C_{l, r}\right)}{\mu\left(S_{r}\right)}$ $=g(l)$. The above theorem is implied by the following.

Lemma 4.2. If there is an $l>0$ such that $\limsup _{r \rightarrow 0} \frac{\mu\left(C_{l, r}\right)}{\mu\left(S_{r}\right)}>0$, then $\underline{R}(x, f) \leq \bar{d}_{\mu}(f)$ on a positive measure set.

Proof. Let us consider a number $l$, a sequence $r_{n} \rightarrow 0$ such that $\lim _{n \rightarrow \infty} \frac{\mu\left(C_{l, r_{n}}\right)}{\mu\left(S_{r_{n}}\right)}>0$ and the sets $T^{-1}\left(C_{l, r_{n}}\right), T^{-2}\left(C_{l, r_{n}}\right), \ldots, T^{-\left\lfloor l\left(\mu\left(S_{r_{n}}\right)^{-1}\right)\right\rfloor}\left(C_{l, r_{n}}\right)$. All these sets are disjoint because if there was $x \in T^{-i}\left(C_{l, r_{n}}\right) \cap T^{-j}\left(C_{l, r_{n}}\right)$ with $i, j \leq\left\lfloor l\left(\mu\left(S_{r_{n}}\right)^{-1}\right)\right\rfloor$, then $\tau\left(T^{\min (i, j)}(x), S_{r_{n}}\right) \leq|i-j|$, and by definition of $C_{l, r_{n}}, T^{\min (i, j)}(x)$ cannot be contained in $C_{l, r_{n}}$, leading to a contradiction. The set $U_{n}=T^{-1}\left(C_{l, r_{n}}\right) \cup$ $T^{-2}\left(C_{l, r_{n}}\right) \cup \ldots \cup T^{-\left\lfloor l\left(\mu\left(S_{r_{n}}\right)^{-1}\right)\right\rfloor}\left(C_{l, r_{n}}\right)$ is then such that

$$
\mu\left(U_{n}\right) \geq\left\lfloor l\left(\mu\left(S_{r_{n}}\right)^{-1}\right)\right\rfloor \mu\left(C_{l, r_{n}}\right) .
$$


Since $\lim _{n \rightarrow \infty} \frac{\mu\left(C_{l, r_{n}}\right)}{\mu\left(S_{r_{n}}\right)}>0$ there is a $c>0$ s.t. $\mu\left(C_{l, r_{n}}\right) \geq c \mu\left(S_{r_{n}}\right)$ for $n$ large enough; then $\mu\left(U_{n}\right) \geq c \frac{\left\lfloor l\left(\mu\left(S_{r_{n}}\right)^{-1}\right)\right\rfloor}{\mu\left(S_{r_{n}}\right)^{-1}}>c^{\prime}>0$. This implies that the set of points

$$
G=\left\{x \in X \text { s.t. } x \in U_{r_{n}} \text { for infinitely many } n\right\}
$$

has positive measure. We remark that if a point $x$ is contained in $U_{r_{n}}$ for some $n$, then $T^{i}(x) \in S_{r_{n}}$ for $i \leq\left\lfloor l\left(\mu\left(S_{r_{n}}\right)^{-1}\right)\right\rfloor$ and then $\tau\left(x, S_{r_{n}}\right) \leq\left\lfloor l\left(\mu\left(S_{r_{n}}\right)^{-1}\right)\right\rfloor$.

Let us denote $\bar{d}_{\mu}(f)=d$; then for each $\delta>0$, if $n$ is large enough, $\mu\left(S_{r_{n}}\right) \geq$ $r_{n}{ }^{d+\delta}$. Hence $\tau\left(x, S_{r_{n}}\right) \leq\left\lfloor l\left(\mu\left(S_{r_{n}}\right)^{-1}\right)\right\rfloor \leq\left\lfloor l r_{n}{ }^{-(d+\delta)\rfloor}\right.$. If $x \in G$, then $\frac{-\log \left(\tau\left(x, S_{r_{n}}\right)\right)}{\log \left(r_{n}\right)} \leq d+\delta$ for infinitely many $n$; then $\underline{R}(x, f) \leq d$. Since $G$ has positive measure we have the following statement.

Remark 4.3. The mixing system without logarithm law given in 7 has a trivial return limit statistic in each centered sequence of balls. This gives an example of a smooth mixing system with trivial limit return time statistics.

\section{REFERENCES}

[1] Barreira, L., Saussol, B., Hausdorff dimension of measures via Poincaré recurrence, Commun. Math. Phys. 219 (2001), 443-463. MR1833809 (2002c:37035)

[2] Chernov, N., Kleinbock, D., Dynamical Borel-Cantelli lemmas for Gibbs measures, Israel Journal of Mathematics, 122 (2001), 1-27. MR.1826488 (2002h:37003)

[3] Dolgopyat, D., Limit theorems for partially hyperbolic systems, Trans. Amer. Math. Soc. 356 (2004), 1637-1689. MR2034323 (2005k:37053)

[4] Degli Esposti, M., Galatolo, S., Recurrence near given sets and the complexity of the Casati-Prosen map, Chaos, Solitons and Fractals 23, no. 4 (2005), 1275-1284. MR2097708 (2006g:37069)

[5] Freitas, A.C.M., Freitas, J.M., Todd, M., Hitting time statistics and extreme value theory, Prob. Th. and Related Fields, to appear.

[6] Galatolo, S., Dimension and hitting time in rapidly mixing systems, Math. Res. Lett. 14, no. 5 (2007), 797-805. MR2350125|(2008i:37007)

[7] Galatolo, S., Peterlongo, P., Long hitting time, slow decay of correlations and arithmetical properties, preprint arXiv:0801.3109.

[8] Galatolo, S., Dimension via waiting time and recurrence, Math. Res. Lett. 12 (2005), 377386. MR2150891 (2006b:37041)

[9] Galatolo, S., Hitting time and dimension in axiom A systems, generic interval exchanges and an application to Birkoff sums, J. Stat. Phys. 123 (2006), 111-124. MR2225238(2007i:37059)

[10] Galatolo, S., Kim, D.H., The dynamical Borel-Cantelli lemma and the waiting time problems, Indag. Math. 18, no. 3 (2007), 421-435. MR2373690(2009b:37005)

[11] Gorodnik, A., Shah, A.N., Khinchin theorem for integral points on quadratic varieties, preprint arXiv:0804.3530, 2008.

[12] Hill, R., Velani, S., The ergodic theory of shrinking targets, Inv. Math. 119 (1995), 175-198. MR1309976 (96e:58088)

[13] Kim, D.H. and Seo, B.K., The waiting time for irrational rotations, Nonlinearity 16 (2003), 1861-1868. MR.1999584 (2004g:37050)

[14] Kleinbock, D.Y., Margulis, G.A., Logarithm laws for flows on homogeneous spaces, Inv. Math. 138 (1999), 451-494. MR1719827 (2001i:37046)

[15] Kim, D.H., Marmi, S., The recurrence time for interval exchange maps, Nonlinearity 21 (2008), 2201-2210. MR2430668 (2009f:37041)

[16] Lacroix, Y., Haydn, N., Vaienti, S., Hitting and return times in ergodic dynamical systems, Ann. Probab. 33, no. 5 (2005), 2043-2050. MR2165587 (2006i:37006)

[17] Liverani, C., On contact Anosov flows, Ann. of Math. (2) 159, no. 3 (2004), 1275-1312. MR2113022 (2005k:37048)

[18] Maucourant, F., Dynamical Borel-Cantelli Lemma for hyperbolic spaces, Israel Journal of Mathematics 152 (2006), 143-155. MR2214457(2008e:37028) 
[19] Pesin, Y., Dimension theory in dynamical systems, Chicago Lectures in Mathematics, University of Chicago Press, 1997. MR 1489237 (99b:58003)

[20] Rousseau, J., Saussol, B., Poincaré recurrence for observations, preprint arXiv:0807.0970.

[21] Sullivan, D., Disjoint spheres, approximation by imaginary quadratic numbers, and the logarithm law for geodesics, Acta Mathematica 149 (1982), 215-237. MR688349 (84j:58097)

Dipartimento di Matematica Applicata, Universita di Pisa, via Buonarroti 1, Pisa, ITALY

E-mail address: s.galatolo@docenti.ing.unipi.it 